\title{
Treatment of Chemotherapy-Induced Subungual Pyogenic Granuloma with Topical Timolol Solution
}

\author{
Natalie M. Williams Brian W. Morrison
}

Dr. Phillip Frost Department of Dermatology and Cutaneous Surgery, University of Miami Miller School of Medicine, Miami, FL, USA

\section{Established Facts}

- Subungual pyogenic granuloma (PG) is commonly triggered by antitumor medications.

- There are favorable reports on the use of topical $\beta$-blocking medications for the treatment of PG.

\section{Novel Insights}

- Topical timolol solution may be an effective treatment for subungual PG.

- This treatment option may be particularly beneficial to individuals prone to multiple peri/subungual PGs, such as those receiving chemotherapy.

\section{Keywords}

Nail pathology $\cdot$ Pyogenic granuloma $\cdot$ Tumor

\begin{abstract}
Introduction: Subungual pyogenic granuloma (PG) can be caused by numerous medications including chemotherapeutic agents. These lesions grow rapidly, oftentimes causing significant pain and bleeding, prompting patients to seek treatment. The management of subungual PG ranges from topical steroids to surgical excision. However, patients with chemotherapy-induced PGs are prone to developing multiple or recurrent lesions. Therefore, finding a therapeutic option that effectively eliminates the tumor and prevents the need for repeated procedural interventions is important. The use of topical $\beta$-adrenergic antagonists has been reported to be effective in regressing cutaneous PG. Case Presen-
\end{abstract}

tation: In this report, we present a case of chemotherapyinduced subungual PG of the toenail arising in a 62-year-old woman that was successfully treated with topical timolol solution. Conclusion: This case highlights the promising use of timolol solution for therapeutically challenging PGs, such as those of subungual regions. This option may be particularly useful for individuals who are vulnerable to multiple PGs secondary to chemotherapy who wish to avoid repeated procedural interventions.

(C) 2020 S. Karger AG, Basel

\section{Introduction}

Pyogenic granuloma (PG) is a relatively common vascular tumor that is characterized by rapid growth of an erythematous, dome-shaped papule with a friable sur- 
Fig. 1. Toenail of left hallux with red, friable papule localized to the distal subungual surface.

Fig. 2. Dermoscopic view of toenail of left hallux demonstrating reddish-white homogenous and structureless areas whose colors vary from completely red to red with whitish zones beneath a thickened nail plate.
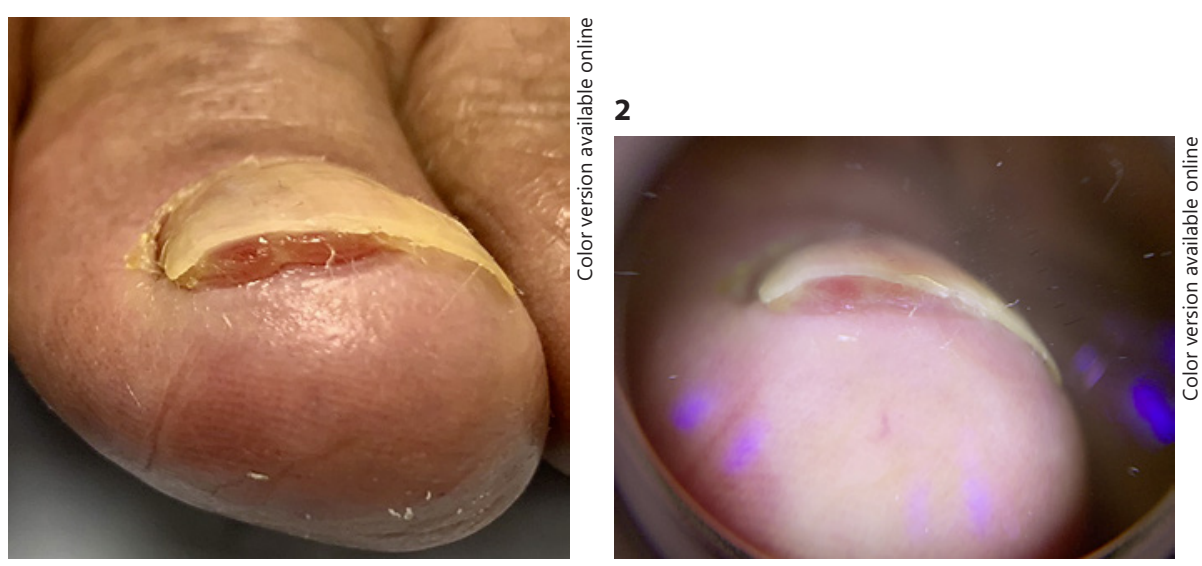

face. It typically arises on normal-appearing skin, especially acral surfaces, or mucosal regions such as the oronasal mucosa. PGs can also develop around or under the nails, which are then referred to as periungual or subungual PGs, respectively. Subungual PGs can be triggered by trauma, peripheral nerve injury, or various medications, including systemic retinoids, highly active antiretroviral therapy, and antitumor agents, such as epidermal growth factor receptor inhibitors, docetaxel, and capecitabine [1]. At a molecular level, the vessel proliferation observed in subungual PGs is due to an imbalance between angiogenesis enhancers and inhibitors [2]. Treatment of subungual PGs largely depends on the etiology and patient preference. Clinicians often begin with a conservative approach, such as prescribing topical medications, but may ultimately recommend more invasive, surgical treatments for resistant lesions. Given the pathogenesis of the lesion, agents that modulate vessel constriction and angiogenesis, such as $\beta$-blockers, may have a role in treating subungual PG.

\section{Case Report}

We present a 62-year-old woman with a history of metastatic breast cancer undergoing treatment with capecitabine for 9 months who presented to our dermatology clinic for evaluation of nail changes. She described generalized redness around the nailbeds of her toenails that had been present for several months, in addition to a red, painful growth beneath the nail of the left big toe that caused nail lifting. She described the pain as a 10/10 in severity and had difficulty ambulating due to it. On physical examination, bilateral halluces demonstrated onycholysis with mild erythema of the nail folds. The toenail of the left hallux demonstrated a red, friable papule localized to the distal subungual surface (shown in Fig. 1, 2).

Timolol for Subungual Pyogenic Granuloma
Given the clinical presentation and history of ongoing chemotherapy, a presumptive diagnosis of drug-induced PG and associated paronychia was given. The patient was prescribed triamcinolone $0.1 \%$ ointment to be applied around the nail folds twice a day and ciclopirox $1 \%$ solution for the nail and surrounding skin nightly. The patient was also encouraged to continue vinegar soaks for 15 min daily. After 1 month, she noted improvement of her nail fold erythema and pain; however, the PG persisted and continued to cause onycholysis. At this point, timolol $0.5 \%$ ophthalmic solution was added to her regimen, and she applied the drops under her nails twice a day for 10 weeks. At 10 -week follow up, the subungual PGs had regressed with only mild distal onycholysis present.

\section{Discussion}

Patients with subungual PGs often seek medical care urgently, as the rapid growth, bleeding, and occasional pain can be anxiety provoking. Histologic confirmation of solitary PGs or lesions without conventional predisposing factors is necessary to rule out malignancy, such as squamous cell carcinoma or amelanotic melanoma. While drug-induced subungual PGs often regress after discontinuation of the offending medication, drug discontinuation was not an option in our case [1]. Conservative treatment of PGs typically includes a short course of high-potency topical steroids and topical antibiotics like mupirocin ointment. If ineffective, patients may undergo surgical curettage under local anesthesia.

In the past decade, topical nonselective $\beta$-adrenergic antagonists, such as timolol or propranolol, have been investigated for the treatment of PGs due to their ability to stimulate peripheral vasoconstriction and decrease angiogenic factors [3]. Like other nonselective $\beta$-antagonists, timolol blocks the action of the sympathetic nervous system by binding to both $\beta_{1}$ and $\beta_{2}$ receptor sites. Further- 
more, various vascular tumors, including PGs, have been shown to express $\beta$-adrenergic receptors [4]. However, it is unclear whether $\beta$-receptor expression predicts response to $\beta$-blocker therapy. Various case series have reported positive results on the use of topical timolol for cutaneous PGs in adults and children. In a series of 6 children $\leq 8$ years old with PG, timolol $0.5 \%$ solution applied 2 to 3 times a day led to at least a partial response without side effects in all patients by 2 months [5]. Another study of 10 individuals $\geq 15$ years old found that 7 patients had at least a partial response within 2.5 months [6]. Although both studies exhibit promising results, their differences suggest that the efficacy of topical timolol for PG may depend on patient age and other factors.

While the literature on the use of timolol for PG of the skin is limited, its use in peri/subungual regions is even scarcer. In 2015, Rosenstein et al. [7] described a patient with recurrent, periungual PGs of the halluces and 1 finger that improved with topical timolol maleate drops. However, subsequent treatment with $0.5 \%$ timolol maleate gel under occlusion was found to be even more effective.

The use of topical $\beta$-blocking medications for subungual PGs was assessed in a series of 10 adult patients with peri/subungual PGs [8]. In this study, topical propranolol $1 \%$ cream was found to effectively treat PGs of the fingernails, including those localized to the nail bed and secondary to chemotherapy. Interestingly, this medication did not improve PGs of the toenails and actually increased exudation and pain in 2 patients prompting them to discontinue the medication.

More recently, a study that included 3 patients with chemotherapy-induced PGs of the toenails reported favorable outcomes with topical timolol [9]. All patients experienced complete resolution with the application of timolol $0.5 \%$ gel twice daily under occlusion for 1 month. Of note, the lesions in this series exhibited a periungual, and not subungual, distribution.

While timolol gel has successfully removed toenail PGs, the utility of timolol solution for subungual PGs has not been described in the literature. In our report, we demonstrate that timolol drops have the potential to completely regress subungual PG of the toenail within 2.5 months. Moreover, we did not require occlusion for this result. This formulation of timolol $(0.5 \%$ ophthalmic drops) can be easily applied to nail lesions, especially those of subungual areas. Therefore, this finding has implications for the management of PG developing in this location.
Overall, this case highlights the promising use of timolol solution for PGs of therapeutically challenging sites, such as subungual regions. This option may be particularly useful for individuals who are vulnerable to multiple PGs secondary to chemotherapy who wish to avoid repeated procedural interventions. Ultimately, larger prospective studies are required to elucidate the efficacy of this medication for PGs, with stratification by medication (e.g., formulation) and lesion features (e.g., location) needed to provide specific recommendations.

\section{Statement of Ethics}

This report was published ethically and in accordance with the World Medical Association Declaration of Helsinki. The patient provided written informed consent to publish photos and the details of the case.

\section{Conflict of Interest Statement}

The authors have no conflicts of interest to declare.

\section{Funding Sources}

The authors did not receive any funding.

\section{Author Contributions}

\section{Author: Natalie M. Williams, BS}

- Substantial contributions to the conception or design of the work or the acquisition, analysis, or interpretation of data for the work

- Drafting the work or revising it critically for important intellectual content

- Final approval of the version to be published

- Agreement to be accountable for all aspects of the work in ensuring that questions related to the accuracy or integrity of any part of the work are appropriately investigated and resolved

- Responsible for writing manuscript

\section{Author: Brian Morrison, MD}

- Substantial contributions to the conception or design of the work or the acquisition, analysis, or interpretation of data for the work

- Drafting the work or revising it critically for important intellectual content

- Final approval of the version to be published

- Agreement to be accountable for all aspects of the work in ensuring that questions related to the accuracy or integrity of any part of the work are appropriately investigated and resolved

- Participated in writing and technical editing of manuscript.
Williams/Morrison 


\section{References}

1 Piraccini BM, Bellavista S, Misciali C, Tosti A, de Berker D, Richert B. Periungual and subungual pyogenic granuloma. Br J Dermatol. 2010;163(5):941-53.

2 Yuan K, Jin YT, Lin MT. The detection and comparison of angiogenesis-associated factors in pyogenic granuloma by immunohistochemistry. J Periodontol. 2000;71(5):701-9.

3 Greenberger S, Bischoff J. Infantile hemangioma-mechanism(s) of drug action on a vascular tumor. Cold Spring Harb Perspect Med. 2011;1(1):a006460.
4 Chisholm KM, Chang KW, Truong MT, Kwok S, West RB, Heerema-McKenney AE. $\beta$-Adrenergic receptor expression in vascular tumors. Mod Pathol. 2012;25(11):1446-51.

5 Wine Lee L, Goff KL, Lam JM, Low DW, Yan AC, Castelo-Soccio L. Treatment of pediatric pyogenic granulomas using $\beta$-adrenergic receptor antagonists. Pediatr Dermatol. 2014; 31(2):203-7.

6 Gupta D, Singh N, Thappa DM. Is timolol an effective treatment for pyogenic granuloma? Int J Dermatol. 2016;55(5):592-5.
7 Resenstein R, Lewellis S, Leger M. Periungual pyogenic granuloma formation in a patient with complex regional pain syndrome. Dermatol Online J. 2015;21(12).

8 Piraccini BM, Alessandrini A, Dika E, Starace M, Patrizi A, Neri I. Topical propranolol 1\% cream for pyogenic granulomas of the nail: open-label study in 10 patients. J Eur Acad Dermatol Venereol. 2016;30(5):901-2.

9 Cubiro X, Planas-Ciudad S, Garcia-Muret MP, Puig L. Topical timolol for paronychia and pseudopyogenic granuloma in patients treated with epidermal growth factor receptor inhibitors and capecitabine. JAMA Dermatol. 2018;154(1):99-100. 